\title{
Marcas blancas, valor percibido y la lealtad en el sector retail
}

\author{
White brands, perceived value and loyalty in the retail
}

sector

\author{
Evelyn Márquez-Peñaloza \\ Universidad Técnica de Machala \\ emarquez2@utmachala.edu.ed \\ Machala - Ecuador \\ Gissella Leiva-Riofrio \\ Universidad Técnica de Machala \\ gleiva1@utmachala.edu.ed \\ Machala - Ecuador \\ Joyce Shagui-González
Universidad Técnica de Machala jshagui1@utmachala.edu.ed \\ Machala - Ecuador \\ Irene Sánchez-González \\ Universidad Técnica de Machala \\ isanchez@utmachala.edu.ec \\ Machala - Ecuador
}

\section{Resumen}

Analizar el valor percibido del consumidor de marcas blancas y su influencia en lalealtad y la construcción del capital de marca en el sector retail. Método descriptivo de enfoque transversal, se aplica una encuesta estructurada auna muestra de 138 consumidores de tiendas departamentales. El valor percibido del consumidor sobre las marcas blancas, influye positivamente sobre la lealtad y el capital de marca. Los minoristas que involucran estrategias de marketing que comuniquen elcompromiso del minorista, tienen resultados positivos en el capital de marca

Palabras claves: Marcas blancas, Marketing, Lealtad, Valor percibido y Capital de marca. 


\begin{abstract}
Analyze the perceived value of the white brands consumer and its influence onloyalty and the construction of brand capital in the retail sector. Descriptive method with a transversal approach, a structured survey is applied to asample of 138 department store consumers. The perceived value of the consumer on white brands, positively influences brandloyalty and capital. Retailers that engage in marketing strategies that communicate retailerengagement have positive results on brand equity.
\end{abstract}

Keywords: White brands, Marketing, Loyalty, Perceived Value and Brand Capital.

\title{
Introducción
}

Las marcas blancas (desde aquí en adelante MB) surgieron en la segunda guerra mundialcon la denominación de $\mathrm{MB}$, estas se caracterizaban por tener envases con diseñossencillos, convirtiéndose en una alternativa de consumo en ese tiempo. Después, en los años 70 estas marcas fueron vistas como una oportunidad de negocio, conocidas ahora como MB. (Zumba, 2015)

Los orígenes de la MB surgen a mediados de 1975 (Sarmiento, 2011), aunque se llamaban productos libres, eran productos de alimentación de compra frecuente, por ejemplo, la leche, las legumbres, el arroz o las galletas, entre otros. Después, se ampliaron a productos de limpieza del hogar o de higiene corporal. Por otra parte, de manera estética, el color del embalaje que se le aplicaba era el blanco. Además, eran asépticos, es decir, no generaban enel cliente grandes emociones a la hora de elegir qué producto comprar. (Gutiérrez, 2020).

Cabe destacar que, a pesar de que las MB son fabricadas por cualquier ente industrial o fabricante, el encargado de todas las actividades del marketing, su merchandising, precio, presentación entre otras, son quienes lo distribuyen empleando así su esfuerzo con el fin de posicionar la marca que los identifica dentro de los procesos y estrategias comerciales adoptados por el distribuidor. (Quiceno, 2020).

Este movimiento nació de la necesidad de la intervención directa de los distribuidores en los productos que vendían. Al estar en contacto directo con los consumidores, veían más de cerca las necesidades y exigencias de estos, y bajo los productos de los que eran únicamente distribuidores no tenían ninguna capacidad de decisión (Sánchez-González et al., 2020). Es decir, al no ser ellos los propios fabricantes del producto, aun conociendo más que los fabricantes que quieren o necesitan los clientes, los distribuidores no tenían métodos o posibilidades de actuación sobre los productos. A raíz de esto, se decantaron por crear una marca propia que buscaba una variedad o serie de productos adaptada a sus clientes. Y, a través de la creación de esta marca propia, también demostraron la implicación con el cliente y la voluntad de entablar entre ellos una relación más directa y cercana (Gutiérrez, 2020). 
En el sector minorista, los productos que comercializan los formatos de supermercados específicamente, bajo el nombre de $\mathrm{MB}$, se caracterizan como productos que se producen y son ofrecidos por el comercio minorista bajo su mismo nombre y marca, por lo tanto, forman una categoría de marca específica. (Valaskova et al., 2018)

Se conoce como MB a los productos que ofrecen las cadenas de supermercados con su nombre de establecimiento sin especificar el nombre del fabricante, vendiendo a un precio inferior que las marcas líderes. Los supermercados con MB por lo general no suelen hacer mucha publicidad, debido a que se encuentran ubicadas cerca de las marcas líderes.

El surtido que venden las cadenas minoristas como sus productos de MB suele ser de un precio más bajo que el resto del surtido en la venta al por menor. Por lo tanto, los clientes conrespuestas más sensibles a los cambios en los precios o en sus ingresos (Horáková, 2015) generalmente están más interesados en comprar este tipo de producto. (Valaskova et al., 2018)

En este contexto, las marcas blancas se han impuesto en los últimos años, llegando a representar actualmente en torno a un $40 \%$ del mercado total. En el ámbito europeo, Españaes el tercer país con mayor penetración de marcas blancas y el primero en términos de volumen. Además, las marcas blancas están aumentando su cuota de mercado frente a las marcas de fabricante en un $80 \%$ de las categorías. (ARAL, 2020)

En Ecuador la comercialización con $\mathrm{MB}$ se desarrolla principalmente en los grandes supermercados como Supermaxi, Akí, Mi Comisariato y Tiendas Industriales Asociadas(TIA), aunque se las llama de distintas maneras (blancas, marcas de distribución, marca propia o marca privada), tienen un objetivo principal para los autoservicios, que es el de aumentar el valor de su negocio al utilizar y combinar tres elementos estratégicos a su favor: información de primera mano del comportamiento del consumidor final, poder de negociación con proveedores, y discrecionalidad en el manejo de la oferta en su cadena. (Superintendencia de Control del Poder de Mercado, 2015)

Hace tres décadas, en Ecuador, esta forma de hacer negocio la empezaron los supermercados (como Mi Comisariato y Supermaxi) vendiendo alimentos de productores terceros bajo su propio nombre, pero ha seguido su expansión hacia otras áreas de consumo, como el mercado de belleza. (Zumba, 2015). Existe una gran oportunidad de desarrollo para los retailers donde Supermaxi y Mi Comisariato son los referentes de las MB en el sector retail ecuatoriano. (Sasson et al., 2017)

Después de una época de gran desempeño, las MB, también conocidas como marcas de distribuidor (MD), frenaron su crecimiento al cierre del 2016 en el mercado ecuatoriano. Enel periodo 2013-2015 lograron capitalizar 8 puntos de penetración, equivalente a doscientos mil hogares ecuatorianos. Siendo el mayor número de compradores a la marca, dieron paso a un rendimiento exponencial, creciendo al doble dígito de los valores anteriores. (Sasson etal., 2017)

En el Ecuador, las pastas destacan como la categoría con mayor presencia, llegando a 4 de cada 10 hogares bajo la modalidad de MB; seguidas por papel higiénico, servilletas y pan. (Sasson et al., 2017) 
En cuanto a facturación se refiere, el papel higiénico se posiciona como el líder dentro del segmento facturando más de US \$13 millones de dólares, afectando a los principales 'players' durante el 2016. El detergente aparece en el TOP 3 de facturación, representando el 5\% a total categoría de detergentes, creciendo a un ritmo del $13 \%$ en valor. Por su parte, 4 decada 10 dólares de las MB se realizan en Guayaquil, posicionándose como el shopper con mayor fidelidad a la marca. (Sasson et al., 2017)

En los últimos años se ha observado un incremento del $46 \%$ en artículos con MB dentro de las cadenas de supermercados Supermaxi y Tía. Las MB gozan de buena aceptación en el mercado ecuatoriano, la preferencia que el consumidor demuestra por estos productos van en aumento, cada año se incrementa en un 7\% en la aceptación de las MB en comparación con elaño anterior. Estas cadenas buscan ofrecerle al cliente un ahorro del 15 al $20 \%$ en sus compras, ofertan productos a menor precio y de excelente calidad, crean alianzas con proveedores que posean altos estándares de calidad, que cumplan con los registros legales, lastécnicas de etiquetados y las Normas INEN. (Andrade-Acero et al., 2019)

Las MB además de ser una de las marcas más vendidas en Ecuador, han llegado a obtener el 3,6 $\%$ de la participación en valor de América Latina, ganando silenciosamente cuota en el mercado, según estudios revelan que el consumidor latino ha modificado su percepción en la elección de las marcas que adquiere teniendo como prioridad las MB con el fin de ahorrar y economizar a la hora de realizar sus compras. (Garavaglia, 2018)

Las MB han llegado abarcar gran segmento del mercado ecuatoriano debido a la variedad de productos que ofrecen con la finalidad de satisfacer las necesidades básicas de los consumidores. Las cadenas de supermercados han despertado en el consumidor el interés de adquirir productos de $\mathrm{MB}$, permitiéndoles a pequeños y grandes productores ecuatorianos generar más plazas de trabajo a nivel nacional, debido a que aproximadamente un $90 \%$ de los fabricantes son nacionales (Bustos, 2016).

El objetivo de esta investigación es ofrecer información importante sobre el comportamiento de los consumidores sobre las MB mediante una descripción completa y una investigación concisa, ya que en Ecuador como en otros países las MB cada vez son más reconocidas y fortalecen su identidad como marca.

\section{Marco Teórico}

\section{Valor percibido}

El valor percibido de la marca es la percepción o la imagen que tiene el consumidor sobre una marca o un producto de una marca (Sánchez et al., 2020), que se genera por todos aquellos atributos que tiene, tanto tangibles (por ejemplo, funcionales) como intangibles (por ejemplo, emocionales). Los cuales, producen en el consumidor de manera subjetiva, una vinculación entre la opinión que tienen sobre el producto, la marca y las expectativas que espera del mismo con la experiencia que ha tenido al consumirlo (Keller, 1993; Leone et al., 2006). Por lo que, el 
resultado puede ser que se produzcan percepciones positivas $\mathrm{y}$, por lotanto, el consumidor sienta la marca valiosa y beneficiosa, la cual deriva a la lealtad hacia la marca o, por lo contrario, se produzcan percepciones negativas. (Gutiérrez, 2020)

El estudio del valor percibido ha ido cobrando importancia con el paso de los años, no solo por la relevancia del concepto en sí mismo, que es considerado como un imperativo estratégico necesario para aquellas empresas que desean mantener su importancia (Sweeney et al., 2001) sino también por la relación influyente que tiene sobre otras variables, entre las que encontramos la calidad percibida y la satisfacción (Cronin et al., 2000). En el ámbito del marketing la investigación sobre el concepto de valor percibido destaca sobre la investigaciónrealizada en torno a otros aspectos como el precio, la estrategia y el comportamiento del consumidor (Gil et al., 2006). (Alguacil, 2017).

Los consumidores comprarán productos que puedan satisfacer sus necesidades y proporcionarles un mayor valor percibido tanto racional como emocionalmente. Las expectativas también deben tenerse en cuenta aquí, porque las percepciones de los consumidores de recibir más o menos de lo esperado variarán según las expectativas. Esto es importante cuando una empresa quiere introducir nuevos productos en el mercado porque debe ser capaz de gestionar bien estas expectativas para que no se conviertan en elementos contraproducentes. (Escobar et al., 2014)

No solamente se compra productos a un buen precio, sino que también se compran beneficiosy valores a cambio de un coste (Sánchez-González et al, 2015). Entonces, ¿cuál debería ser el precio de nuestros productos? Bueno, dependerá de varios factores, desde el costo de producción hasta el precio que creemos que pagará la gente, comprendiendo los beneficios que posee el producto. Obviamente, su precio debería mantener una cierta brecha entre el precio de los productos competidores y el costo de producción. Si lo vendemos demasiado caro, tendremos menos clientes capaces de comprarlo, y si es muy barato, casi no tendremos ganancia, por lo que debemos buscar un equilibrio, es decir, sabiendo que además de muy poca ganancia, el precio es demasiado. La baja también se relaciona con la mala calidad del producto (Escobar et al., 2014).

H1: Los productos de las MB podrían no tener una buena calidad percibida por parte del consumidor en la relación calidad - precio del producto.

\section{Lealtad}

La importancia que tienen las MB en el momento de la compra y consumo, es que se involucran emociones, experiencias y deseos convirtiéndose en una fidelidad de marca. En este orden de ideas, hay que aclarar que factores como, la situación económica mundial, la globalización, la liberación de los mercados y los avances tecnológicos, trajeron consigo efectos, como el aumento de la competencia, lo cual provocó una saturaciónde los mercados, obligando de alguna manera a que las empresas realizar estrategias de satisfacción de clientes, para establecer relaciones duraderas, y así de esta manera se originóel concepto de fidelidad (Rodríguez, 2017). 
Con base en esto, la fidelidad se puede evaluar desde, a) la fidelidad comportamental, en donde se asocia la frecuencia de compra y la recompra; b) La fidelidad afectiva, en donde el consumidor crea un vínculo emocional con la marca o muestra una actitud positiva hacia determinada marca y c) la fidelidad cognitiva, que se refiere a la atención y concentración que el consumidor presta en el momento de la decisión de compra de una marca (Jacoby et al., 1973). (Rodríguez, 2017)

También se puede decir que la lealtad a la marca tiene relación con la lealtad que tienen los consumidores hacia el minorista y su influencia en la intención de compra, por lo que, la imagen favorable de la marca que tiene el minorista, es el precio de los productos y por la calidad que los consumidores perciben, tiene mucha influencia en la lealtad hacia las MB. (Rodríguez, 2017) $\mathrm{H} 2$ : La conciencia de precio tiene un efecto positivo sobre la lealtad actitudinal a las marcas blancas. (Gómez et al., 2016)

\section{Capital de marca}

Según (Kotler, 2012), el capital de marca o Brand Equity es el valor añadido de que se dota a productos y servicios. Este valor se refleja en cómo piensan, sienten y actúan los consumidores respecto a la marca, o en los precios, la participación del mercado y la rentabilidad que genera la marca para la empresa (Sánchez et al., 2020).

Los enfoques centrados en el consumidor, estudian el brand equity desde la perspectiva de los consumidores, ya sean individuos u organizaciones. La premisa de este modelo es que los consumidores ven, leen, oyen, aprenden, piensan y sienten con respecto a la marca a lo largo del tiempo. En otras palabras, el poder de una marca reside en la mente de los clientes realeso potenciales y en sus experiencias directas e indirectas con ella (Clendenes, et al., 2017).

El precio juega un papel fundamental en la configuración de las MB "propuesta (Richardson, et al.,1996), ya que las MB se caracterizan por un posicionamiento de precios bajos que proviene de los consumidores de la forma que perciben las $\mathrm{MB}$ como un precio conveniente en comparación con las marcas del fabricante (Ailawadi, et al., 2008). El posicionamiento inicial de las MB se basó en ofrecer a los consumidores un precio competitivo como alternativa a marcas de fabricantes, ayudando a los minoristas a competir en el segmento sensible a los precios (Corstjens et al., 2000).

Bao (2011) describió que los compradores creen que las MB generalmente tienen precios bajos y por ende baja calidad. Sin embargo, estas marcas tienen una buena relación entre calidadprecio. Además, recientemente, las MB se han desarrollado con una mayor orientación hacia la calidad, ofreciendo una propuesta de valor similar a las marcas del fabricante (Hoch et al., 1993). (Porral, et al., 2016)

Es importante señalar que ha venido evolucionando el posicionamiento de las MB, desde el enfoque en el precio de venta, hasta las propuestas de valor basadas en la calidad. Las investigaciones previas muestran que las MB se conciben inicialmente como una buena opción entre "calidad-precio" para aquellos consumidores con gran sensibilidad de precio (Calvo et al., 2016). 
H3: Las marcas blancas representan una buena alternativa económica para las familias al momento de adquirir productos de consumo.

\section{Materiales y métodos}

En el presente apartado se presentan los métodos que se aplicaron en la investigación para recolectar información de los consumidores en lo que respecta a la determinación de la muestra, elaboración de encuesta con preguntas de opción múltiple y escala likert de 1 a 5 en donde el encuestado manifiesta su nivel de totalmente de acuerdo o desacuerdo según las opciones planteadas para la evaluación de las variables.

La población considerada son los consumidores económicamente activos que adquieren productos de las MB de los cuatro principales supermercados de la ciudad de Machala como lo son Supermaxi, Mi Comisariato, Akí y TÍA S.A, de esta manera se podrá comprobar las hipótesis, en la cual ayudará a determinar el grado de aceptación y el impacto económico que tienen estas MB en las familias.

Además, los supermercados Mi Comisariato, Akí y Supermaxi usan la estrategia de mantener el nombre del establecimiento como $\mathrm{MB}$ en sus distintos productos, las cuales pertenecen a categorías diferentes y se manejan indistintamente dependiendo de la categoría a la que pertenecen, tales como: granos, enlatados, bebidas, embutidos, entre otros, mientras que almacenes Tía S.A. maneja su MB con un nombre diferente al de su logo que es TA'RIKO, estos se caracterizan por ser productos de buena calidad y por tener un precio accesible.

Asimismo, estas empresas siempre buscan obtener nuevos productos, lo cual va de la mano para obtener mayor participación en el mercado a pesar de que empezaron con productos básicos para el consumo en el hogar, cada vez van explorando nuevas categorías ampliando su cartera de productos. Las promociones, el beneficio de sus productos, sus precios bajos y competitivos, han contribuido para que las MB ganen mayor participación en el punto de venta y el mercado en general.

Se realizó una investigación de campo, que se planificó desde el 02 de enero hasta el 23 de febrero del 2019, donde se aplicaron 138 encuestas a los consumidores, siguiendo el método 
probabilístico, donde cada uno de los miembros del equipo de investigación encuestó de manera personal a cada diez consumidores que adquirieron los productos de $\mathrm{MB}$, durante unos 20 minutos.

Para la respectiva investigación se empleó la técnica del muestreo aleatorio simple, para poner en práctica el principio de equiprobabilidad, es decir, que cada persona de la muestra tenga la misma probabilidad de ser elegida para ser investigada.

Para el procesamiento e interpretación de los datos en el caso de las encuestas se utilizó el programa IBM SPSS y Excel. Los resultados obtenidos de las encuestas se dieron a conocer a través de cruces de variables, histogramas, diagramas de barra y gráficas de pastel.

Los resultados recolectados en la encuesta, permitieron identificar el perfil del consumidor de los supermercados de la ciudad de Machala, que son personas económicamente activas entre 25 a 30 años $(42,1 \%)$ y de 31 a 36 años $(31,4 \%)$. Para complementar, los compradores gastanen promedio de $\$ 36$ a 46 (28\%) y más de $\$ 58$ (27\%) en el total de su facturación al realizarlas compras.

\section{Resultados}

En el gráfico 1 muestra el análisis de los resultados arrojados por las encuestas ejecutadas a los consumidores que ingresaban a realizar sus compras en los distintos supermercados, los mismos que son objeto de estudio. El valor superior que indica cual es el supermercado que con más frecuencia los consumidores compran los productos de $\mathrm{MB}$ es de Mi Comisariato con el $(29,71 \%)$ y se mantiene con el supermercado Supermaxi con $(28,99 \%)$, por lo que muestra que dichos supermercados son los predilectos por los consumidores para realizar sus compras.

A continuación, en el gráfico 2 se puede evidenciar que el beneficio que prevalece en los consumidores es el factor precio con $(49,28 \%)$, debido a que estos productos de MB son económicos, por lo que se puede decir que este factor tiene un gran peso para los consumidores al momento de escoger qué productos adquirir.

En el gráfico 3 se han identificado dos resultados muy sobresalientes. El primero de ellos se encuentra neutral $(34,78 \%)$ en lo referente a la lealtad que tienen los consumidores hacia los productos de $\mathrm{MB}$, el segundo resultado también es destacado indicando un gran porcentaje de consumidores $(32,61 \%)$ son leales a las MB ante la elección de qué productos adquirir.

Por último, en el gráfico 4, la variable más importante sobre la información es que los productos de MB brindan un nivel de confianza alto con $(39,13 \%)$, y también dichos productos proporcionan un buen valor adquisitivo en la compra con $(24,64 \%)$ 


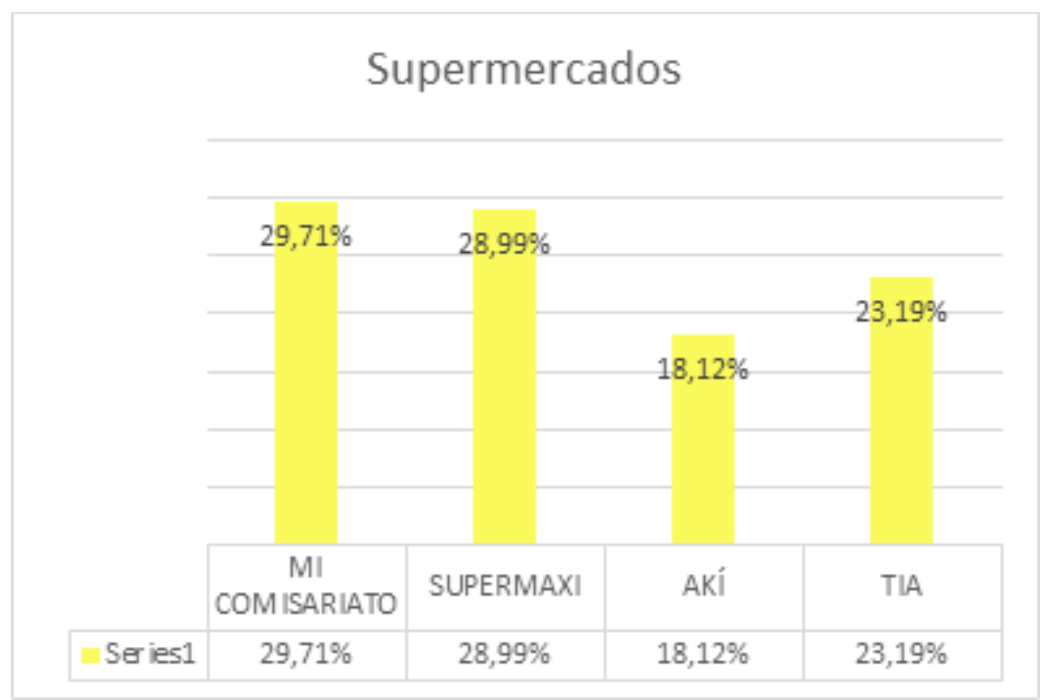

Figura 1.

Compra en supermercados

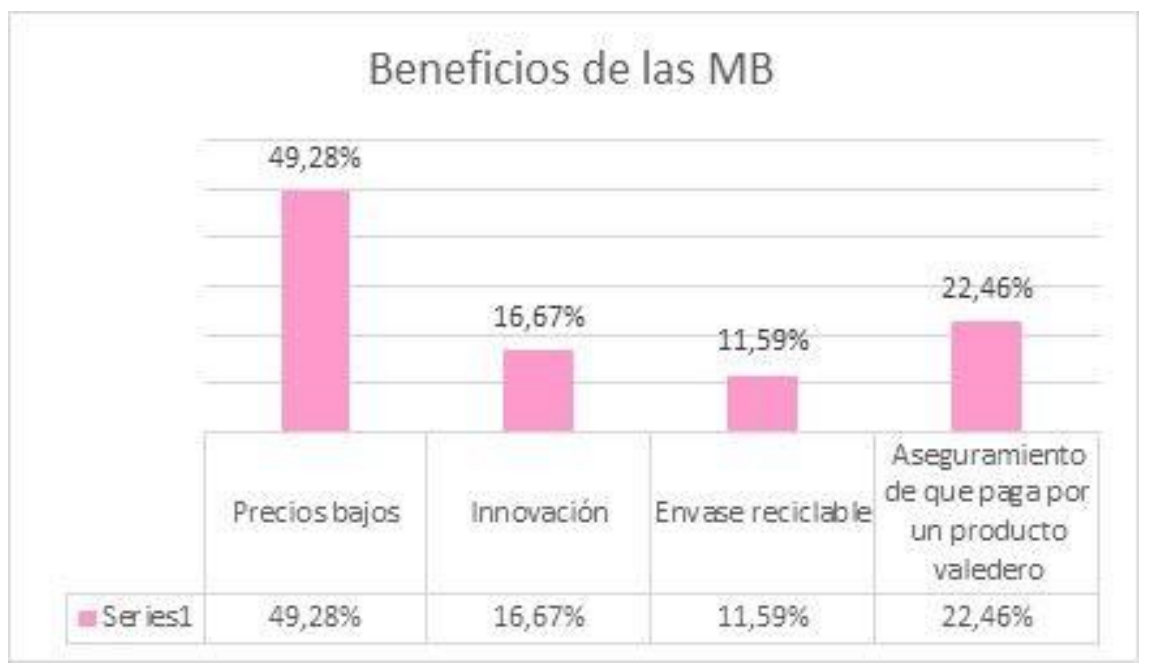

Figura 2.

Beneficios de las MB

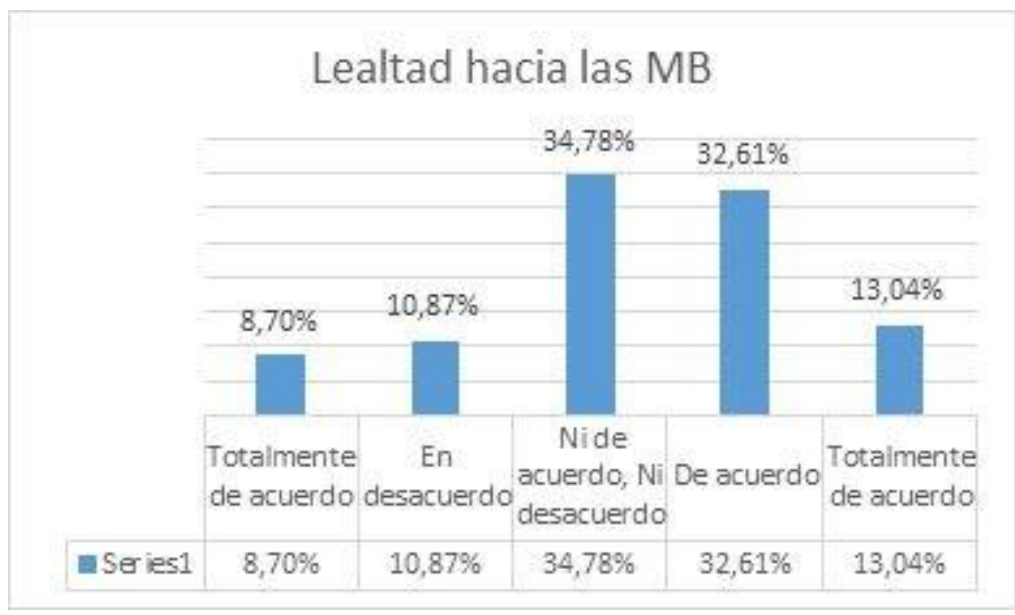

Figura 3.

Lealtad hacia las MB 


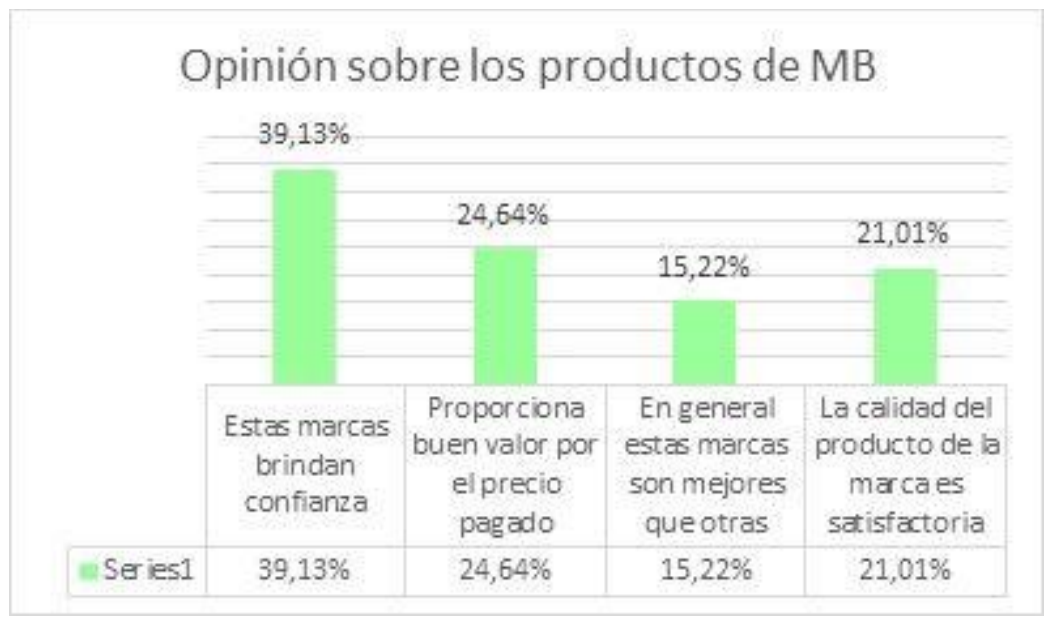

Figura 4.

Opinión sobre los productos de MB

\section{Conclusiones}

El estudio realizado ha aportado en el aumento de conocimiento sobre los productos MBde los supermercados, así como la identificación de qué factores influyen positivamente en la decisión de compra de los consumidores. El contraste de las hipótesis propuestas permite desarrollar las siguientes conclusiones.

En primer lugar, después de analizar el efecto que tienen estas marcas sobre el valor percibido de los consumidores, el presente trabajo muestra que los consumidores tienen una buena imagen sobre los productos de MB, ya que consideran que tienen buena calidad y cumplen adecuadamente sus funciones, por lo que se concluye que el valor percibido posee un efecto positivo en ellos y los supermercados se encargan cada día de fortalecer esta imagen por medio de adecuadas estrategias de marketing, siendo esa iniciativa acogida con buena actitud por parte de los consumidores, y se lo puede constatar en la recompra de los productos.

Además, el estudio pone en manifiesto que la percepción de los consumidores sobre el precio de los productos, influye positivamente en el valor de las MB. Así mismo, para seguir otorgándoles mejores beneficios a los consumidores con estos productos, es recomendable usar la estrategia de valor agregado y ofrecerle al consumidor más beneficios de los que ya sepuede estar otorgando. En la medida en que la propuesta de valor sea singular o muy específica para sus consumidores, más interesados estarán en adquirir dichos productos.

En segundo lugar, el valor percibido también tiene efectos en la lealtad de los consumidores, debido a que es algo difícil de conseguir por parte de los supermercados y depende de algunos factores como el precio y la calidad de éstas MB, ya que los consumidores prefieren la relevancia, las aportaciones que brinda el producto y no necesariamente en el diseño del mismo. Además, la lealtad de los consumidores puede variar según el tipo de producto que desean adquirir y aquí el marketing promocional juega un papel significativo. 
Para mejorar más los niveles de lealtad de los consumidores, es necesario implementar estrategias de fidelización, para superar sus expectativas por medio la recolección de datos, que permitirá a los supermercados del país conocer los hábitos, preferencias y gustos de cada consumidor, para actuar en consecuencia, entregando una experiencia única, personalizada y causar una muy buena impresión, así mismo, ganarse la confianza del consumidor. Además, es fiable recompensar a los consumidores que ya son leales, ofreciéndole obsequios por la cantidad de productos de MB que compren.

En tercer lugar, se reafirma los consumidores prefieren los productos de MB ante otras marcas, y contribuye significativamente al factor de capital de marca, debido a que los consumidores no tienen dificultades en el elegir estas marcas ante otras a pesar de tener características similares, y se puede decir que la lealtad de los consumidores, que los supermercados han logrado sembrar y fortalecer, este representa un papel muy importante en sus decisiones de compra.

Una de las estrategias de marketing que se debe implementar para mantener a los consumidores prefiriendo estás MB es la identidad de marca, ya que esta estrategia facilita el reconocimiento del producto por parte del consumidor, por lo que resulta necesario implementar estrategias de diferenciación del producto, haciendo que la marca se destaque de manera positiva de sus competidores, por su aspecto, utilidad, funciones, calidad, logrando que el comprador prefiera consumir los productos de MB.

Las MB crecen tanto en España como en Ecuador cada día. El motivo de este cambio es que estos productos de $\mathrm{MB}$ suelen resultar más económicos que las marcas líderes, además por las crisis económicas, los consumidores han perdido su poder adquisitivo. Por este motivo comparan precios más que nunca y buscan todo tipo de promociones y ofertas.

Los productos de las MB a pesar de que iniciaron en el mercado con unas pocas líneas de productos, en la actualidad se pueden encontrar estas marcas en todo tipo de categoría, así mismo, los consumidores están más interesados por adquirir estas marcas. A medida que pasa el tiempo y conocen más sobre ellas, su confianza va creciendo y van siendoconsiderados las MB como de buena calidad y buen precio haciendo que los consumidores prefieran adquirir dichos productos de MB.

Por otro lado, el mercado ecuatoriano comercializa las MB desde el año 2006, desdeentonces estas marcas han luchado para tener aceptación en el mercado y de la misma manerapermanecer en la mente del consumidor para obtener valor sobre sus productos. En la actualidad los productos de MB proporcionan una gran competencia ante las marcas líderes y provoca que sean mejor percibidas por los consumidores, permitiéndoles ahorrar desde un 15\% hasta un 35\% dependiendo del producto de esta manera tiene una ventaja ya que estos productos tienen similar característica de las marcas líderes. (Telégrafo, 2012) 
Una de las principales limitaciones del presente estudio es el ámbito geográfico y la posibilidad de evaluar relaciones con otras variables como la publicidad, por lo que se considera una línea futura de investigación.

Este trabajo de investigación es producto del grupo de investigación COMARK y de los estudiantes de $\mathrm{V}$ nivel de Mercadotecnia que ejecutan sus horas de prácticas de servicio comunitario en la asignatura de Marketing Comunitario.

\section{Referencias:}

Ailawadi, K. L., Pauwels, K., y Steenkamp, J. B. (2008). Private label use and store loyalty. Journal of Marketing, 72(6), 19-30. https://doi.org/10.1509/jmkg.72.6.019

Alguacil-Jiménez, M. (2017). Análisis de la percepción de marca y su relación con el rendimiento percibido en servicios deportivos: comparación entre público y privado. Universidad de Valencia, 176. https://dialnet.unirioja.es/servlet/tesis?codigo=157048

Andrade-Acero, N., y León-Campoverde M. (2019). Las marcas blancas vs marcas tradicionales: Estudio comparativo en productos de la canasta básica. Universidad $\begin{array}{lllll}\text { Estatal de } & \text { Milagro, } & 7 & - & 8 .\end{array}$ http://repositorio.unemi.edu.ec/xmlui/handle/123456789/4902

ARAL Revista del gran consumo (2020, julio 17). Las marcas blancas aumentan su cuota de mercado en un $80 \%$ de las categorías. https://www.revistaaral.com/distribucion-conbase-alimentaria/marcas-blancas-aumentan-su-cuota-mercado-en-80categorias_15141912_102.html

Bao, Y., Sheng, S., Bao, Y., y Stewart, D. (2011). Assessing quality perception of private labels: Intransient cues and consumer characteristics. Journal of Consumer Marketing, 28(6), 448-458. https://doi.org/10.1108/07363761111165967

Bustos, B. (2016). Influencia del precio en el proceso de compra de productos de marca distribuidor, en la categoría productos de limpieza de la cadena de supermercados mi comisariato en la ciudad de Guayaquil. Universidad Católica de Santiago de Guayaquil. 25 - 27. http://repositorio.ucsg.edu.ec/handle/3317/5627

Calvo, C., Martínez, V. A., y Juanatey, O. (2016). Influence of manufacturer signature on store brands'loyalty and purchase intention. Revista de Administración de Empresas, 56(1), 29-42. https://doi.org/10.1590/S0034-759020160104

Corstjens, M., y Lal, R. (2000). Building store loyalty through store brands. Journal of Marketing Research, 37(3), 281-291. https://doi.org/10.1509/jmkr.37.3.281.18781 
Clendenes, M., y Guarnizo, J. (2017). Factores que influyen en la compra de marcas blancas por los clientes del supermercado Plaza Vea del Real Plaza en la ciudad de trujillo. $\begin{array}{lllll}\text { Universidad Privada } & \text { Antenor } & \text { Orrego. } & 35 & \end{array}$ https://repositorio.upao.edu.pe/handle/20.500.12759/3466

Cronin, J. J., Brady, M. K., y Hult, G. T. M. (2000). Assessing the effects of quality, value, and customer satisfaction on consumer behavioral intentions in service environments. Journal of retailing, 76(2), 193-218. https://doi.org/10.1016/S0022-4359(00)00028-2

El telégrafo. (2019, julio 28) Las marcas blancas promueven el ahorro en los consumidores. https://www.eltelegrafo.com.ec/noticias/economia/4/las-marcas-blancas-promueven-e 1-ahorro-en-los-consumidores

Escobar, L. V., y Urriago, A. F. (2014). Características del producto y percepción de la marca que determinan la decisión de compra de productos de marcas blancas en las grandes superficies de Cali. Universidad Autónoma de Occidente. 15 - 17. https://red.uao.edu.co/handle/10614/6422

Garavaglia, V. (2018, septiembre 20). El sector FMCG atraviesa un punto de inflexión. Kantar. https://www.kantarworldpanel.com/ar/Noticias/El-sector-FMCG-atraviesa-unpunto-de-inflexin

Gil, I., Sánchez, M., Berenguer, G., y González G, M. (2006). Encuentro de servicio, valor percibido y satisfacción del cliente en la relación entre empresas. Cuadernos de Estudios Empresariales, (15), 47-72. ISSN: 1131-6985

Gómez, M., Rubio, N., y Oubiña, J. (2016). Antecedentes de la lealtad a las marcas de distribuidor diferencias entre grandes y pequeños compradores. Technical Report. 23. https://ideas.repec.org/p/ovr/docfra/1105.html

Gutiérrez, M. (2020). El valor percibido de las marcas del distribuidor y del fabricante: un análisis comparativo de su posicionamiento en los canales de distribución. Universidad $\begin{array}{lllll}\text { Autónoma } & \text { de } & \text { Barcelo. } & 9 & -\end{array}$ https://ddd.uab.cat/pub/tfg/2020/228096/TFG mgutierrezruano.pdf

Hoch, S. J., y Banerji, S. (1993) When do private labels succeed?. Sloan Management, 34-45 http://sloanreview.mit.edu/article/when-do-private-labels-succeed/

Horáková, M. (2015). Consumer Behavior of College Students in the Czech Republic. Ingenta Connect. 7 (4), 68-85. https://doi.org/10.7441/joc.2015.04.05 
Keller-Lane, K. (1993). Conceptualizing, measuring, and managing customer-based brand equity. Journal of Marketing, 57(1), 15-17. https://doi.org/10.2307/1252054

Kotler, P., y Keller, K. (Ed.). (2012). Dirección de Marketing. Evanston: PearsonEducation.

Jacoby, y Kynner. (1973). Brand loyalty vs repeat purchasing behavior. Journal of marketing research, 10(1), 1-9. https://doi.org/10.1177/002224377301000101

Quiceno, E. A. (2020). Impacto de las marcas propias en los supermercados de Copacabana. Tecnológico de Antioquia Institución Universitaria. 21 - 25. https://dspace.tdea.edu.co/handle/tdea/634

Leone, R.P., Luo, A.M., Rao, V.R., Keller, K.L., Mcalister, L. y Srivastava, R. (2006). Linking brand equity to customer equity. Journal of Service Research, 9(2), 125-138, https://doi.org/10.1177/1094670506293563

Richardson, P., Jain, A. K., y Dick, A. S. (1996). Household store brand proneness: A framework. Journal of Retailing, 72(2), 159-185. https://doi.org/10.1016/S00224359(96)90012-3

Rodríguez, D. V. (2017). Fidelidad del consumidor hacia las marcas propias en los canales de distribución. Universidad Santo Tomas. https://repository.usta.edu.co/bitstream/handle/11634/4451/RodriguezDaniela2017.pdf ?sequence $=1 \&$ is Allowed $=\mathrm{y}$

Sánchez-González, I., Benítez-Luzuriaga, F y Arias-Montero, E. (2015). El precio en el marketing. Universidad Técnica Machala. http://repositorio.utmachala.edu.ec/handle/48000/6893

Sánchez-González, I., Gil-Saura, I y Ruiz-Molina, M. (2020). Effects of sustainable development perceived by the consumer. A model proposal for hypermarkets in Ecuador. Estudios Gerenciales, 36(154), 27-42. https://doi.org/10.18046/j.estger.2020.154.3470

Sánchez-González, I., Gil-Saura, I., y Ruiz-Molina, M. (2020). Ethically Minded Consumer Behavior, Retailers' Commitment to Sustainable Development, and Store Equity in Hypermarkets. Sustainability, 12(19), 80-41. https://doi.org/10.3390/su12198041

Sánchez-González, I; Benítez-Luzuriaga, F.; Moscoso-Parra, A. y Muñoz-Suarez, M. (2020). Eldesarrollo sostenible en las mipymes de Ecuador y su impacto en el consumidor. Cumbres 6(1), 33-42. https://doi.org/10.48190/cumbres.v6n1a3 
Sarmiento, C. (2011, abril 05). Historia de las marcas blancas y marcas propias. http://marcasblancas3.blogspot.com

Sasson, J., y Rossi, A. (2017). El consumidor ecuatoriano y su preferencia en marcas propias. Retail Software. https://rp3.com.ec/el-consumidor-ecuatoriano-y-su-preferencia-enmarcas-propias/

Sweeney, J. C., y Soutar, G. N. (2001). Consumer perceived value: The development of a multiple item scale. Journal of retailing, 77(2), 203-220. https://doi.org/10.1016/S0022-4359(01)00041-0

Superintendencia de Control del Poder de Mercado. (2015). Estudio de Mercado Marcas Propias. http://www.scpm.gob.ec/sitio/wp-content/uploads/2019/02/MarcasPropias-Ver sinpblica.pdf

Valaskova, K., Kliestikova, J. y Krizanova, A. (2018). Consumer Perception of Private Label Products: An Empirical Research. Journal of Competitiveness, 10(3), 149-163. https://doi.org/10.7441/joc.2018.03.10

Zumba, L, R. (2015, octubre 19). Las marcas propias, una tendencia que evoluciona. http://www.espol.edu.ec/espol/docs_escribe/3891.pdf 